\title{
LA IMPORTANCIA DEL INTERVALO DE LA FLORACIÓN EN EL MEJORAMIENTO PARA LA RESISTENCIA A SEQUÍA EN MAÍZ TROPICAL ${ }^{1}$
}

\author{
J. Bolaños ${ }^{2}$, G.O. Edmeades ${ }^{2}$
}

\section{COMPENDIO}

La longitud de intervalo entre la aparición de estigmas y antesis se incrementa cuando la sequía coincide con la época de floración del maíz (Zea mays L.). Cuatro poblaciones élite de maíz tropical del CIMMYT están siendo mejoradas para resistencia a sequía por esquemas de selección recurrente ( $\mathrm{S}_{1}$ o hermanos completos) para rendimiento de grano y varias otras características, tanto bajo estrés de sequía, como bajo condiciones de buena humedad. Los datos recolectados de más de 2,000 famillas por población evaluadas en parcelas de un solo surco, bajo tres niveles de estrés de humedad de suelo (1. estrés severo durante el período de pre y postfloración; 2. estrés intermedio durante el llenado de grano; y 3. irrigación normal, todos en ausencia de lluvia), mostraron correlación débil o ausencia de ésta entre el rendimiento de grano y otras características relacionadas al balance hídrico de la planta, tales como: enrollamiento foliar y senescencia, foto-oxidación, concentración foliar de clorofila, tasa de elongación vegetativa, temperatura foliar y potencial hídrico matutino. El rendimiento bajo todos los niveles de estrés fue correlacionado negativamente con el intervalo de floración y el intervalo de floración se incrementó debido a la sequía. Asimismo, los granos y mazorcas por plantase redujeron significativamente. En todas las poblaciones el rendimiento disminuyó aproximadamente $10 \%$ por día de incremento en el intervalo de floración y hasta 8 días. En varias situaciones de estrés, la heredabilidad de sentido amplio para el intervalo de floración fue mayor que aquella del rendimiento de grano y la correlación genética entre rendimiento de grano y el intervalo de floración aproximadamente -1.00. Los sintéticos formados a partir de una de las poblaciones, seguidos de selección bidireccional y evaluados bajo sequía, demostraron ventajas adaptativas como baja temperatura foliar, baja senescencia foliar, un intervalo de floración reducido y hojas erectas, especialmente, cuando todas estas características fueron combinadas en un índice de selección. La selección por intervalo de floración corto y alto rendimiento de grano puede ser un medio eficaz de mejorar la tolerancia a la sequía en maíz tropical.

Palabras clave adicionales: Zea mays, balance hídrico, esteres, sintéticos.

\section{INTRODUCCIÓN}

El propósito de este trabajo ha sido evaluar los distintos criterios de selección posibles a usarse en programas de mejoramiento para resistencia a sequía en maíz tropical. Los datos han sido obtenidos en viveros de selección usados por CIMMYT en México en sus programas de mejoramiento para resistencia a sequía.

Cuatro poblaciones élite de maíz tropical del CIMMYT están siendo mejoradas para resistencia a se-

\begin{abstract}
The length of the Interval between anthesis and silking (ASI) is increased by drought which coincides with flowering. Four elite CIMMYT lowland tropical populations are undergoing recurrent selection $\left(\mathrm{S}_{1}\right.$ or full-sib) for improved grain yield and several other traits under drought and well watered conditions. Data collected from more than 200 families per population grown In single row plots under three water stress levels (pre and post-flowering stress; post-flowering stress; normal irrigation, all in the absence of rain) showed weak or no correlation between grain yield and traits related to plant water status, such as leaf rolling and senescence, photooxidation, leaf chlorophyll concentration, shoot elongation rate, canopy temperature and predawn water potential. Yield under all levels of stress was significantly negatively correlated with AST, and as AST increased due to drought, kernels and ears per plant were significantly reduced. In all populations yield decreased by approximately $10 \%$ per day increase in AST up to 8 days. In several stress situations broad-sense heritability of AST was greater than that of grain yield and the genetic correlation between grain yield and AST approached -1.00 . Synthetics formed from one population following bidirectional selection and tested under drought showed adaptive advantage of cool canopy temperature, delayed leaf senescence, reduced AST and erect leaves, especially when all were combined with grain yield in a single index during selection. Selection for reduced AST and high grain yield under drought can be an effective means of improving drought tolerance in tropical maize.
\end{abstract}

quía en esquemas de selección recurrente. Cerca de 1.000 líneas S1 de cada población se siembran en viveros de observación en el verano en Sonora, México, y se seleccionan las mejores 200-250. Estas se siembran en parcelas de un solo surco de 2.5 metros con dos repeticiones y tres niveles de sequía en la temporada seca en Tlaltizapán, México; y las mejores 50 son seleccionadas para recombinación. Las selecciones se basan en un índice que incorpora rendimiento de grano, sincronización floral y una serie de características indicativas de la capacidad del genotipo de mantener un balance hídrico positivo bajo sequía. Los tres niveles de sequía son: a) Irrigación normal, b) Estrés intermedio o

\footnotetext{
Presentado en la XXXV Reunión Anual del PCCMCA, San Pedro Sula, Honduras. 1989

2 Programa de Maíz, CIMMYT, México.

${ }^{3}$ Publicado en Agronomía Mesoamericana, Vol. 1 (1990).
} 
estrés durante el llenado de grano o período postfloración (el cultivo se riega adecuadamente hasta la floración y completa su ciclo con agua almacenada en el suelo), y c) Estrés severo donde el cultivo solamente recibe riego para la gemunaci6n y su establecimiento y completa su ciclo con agua almacenada en el suelo.

\begin{tabular}{ccccc}
\multicolumn{5}{c}{ Germoplasma bajo mejoramiento para resistencia a } \\
sequía & & \\
Población & Madurez & Color & \multicolumn{1}{c}{ Tipo } & Familia \\
\hline Pool 16 & Precoz & Blanco & Dentado & H.C. \\
Pool 18 & Precoz & Amarillo & Dent/Crist. & S1 \\
La Posta & Tardío & Blanco & Dentado & S1 \\
Pool 26 & Tardío & Amarillo & Dentado & S1
\end{tabular}

\section{Criterios de Selección}

Los criterios de selección son los siguientes:

\footnotetext{
* Rendimiento de grano bajo sequía (incrementar)

* Rendimiento de grano bajo irrigación (mantener)

* Intervalo de la floración bajo sequía (reducir)

* Número de mazorcas por planta bajo sequía (incrementar)

* Enrollamiento foliar bajo sequía (reducir)

* Temperatura foliar bajo sequía (reducir)

* Fogueo foliar bajo todos los niveles (reducir)

* Elongación vegetativa bajo sequía (incrementar)

* Ángulo erecto de la hoja (incrementar)

* Clorofila bajo todos los niveles (incrementar)
}

\section{RESULTADOS Y DISCUSIÓN}

\section{Correlaciones lineales entre los parámetros usados en la selección y rendimiento de grano bajo sequía}

Correlaciones pobres fueron encontradas entre la mayoría de jas características morfológicas y fisiológicas examinadas y el rendimiento de grano bajo sequía en las 4 poblaciones élite de maíz tropical (Cuadro 1A). Estas características morfológicas y fisiológicas son indicativas de la capacidad de mantener un balance hídrico positivo bajo sequía y por tanto tienen supuestamente un valor adaptivo, como la baja temperatura foliar, el enrollamiento foliar, la senescencia foliar y la elongación vegetativa. Sin embargo, el rendimiento de grano bajo sequía solamente correlacionó significativamente $(\mathrm{P}<0.001)$ con el intervalo de la floración y el número de mazorcas por planta para las 4 poblaciones, pero no con ningún parámetro fisiológico o morfológico. El intervalo de la floración se define como el intervalo en tiempo- entre la dehiscencia de polen (antesis) y la extrusión de estigmas para el 50\% de las plantas presentes por cada genotipo (anthesis silking interval, ASI).
Virtualmente, ninguna de tas características morfológicas y fisiológicas examinadas correlacionó significativamente con el intervalo de la floración en las 4 poblaciones (Cuadro $1 \mathrm{~B}$ ). Aunque se sabe que la extrusión de los estigmas es un proceso muy sensible a déficits hídricos, la variabilidad observada entre las líneas en el intervalo de la floración no estuvo correlacionada con otros indicadores del balance hídrico, ni siquiera inclusive con el potencial hídrico matutino de hojas. Esto sugiere que el intervalo de la floración parece depender en factores genéticos de la distribución de la materia seca y no en el balance hídrico de los genotipos.

Cuadro l. Coeficientes de correlación lineal entre varias características morfológicas y (A) rendimiento de grano bajo sequía y (B) el intervalo de floración bajo sequía en 4 poblaciones élite de maíz tropical del CIMMYT. Los datos son de 1987 y 1988 en Tlaltizapán, México.

\begin{tabular}{cccc}
\hline Característica & Pool 16 & Pool 18 & La Posta Pool 26 \\
& 194 fam & 250 fam & 232 fam 235 fam \\
\hline
\end{tabular}

(A) Rendimiento de grano bajo sequía

\begin{tabular}{lcccc} 
Días a antesis & n.s. & -0.411 & -0.338 & -0.398 \\
Mazorcas por planta & 0.701 & 0.695 & 0.783 & 0.805 \\
Intervalo de floraci6n & -0.475 & -0.515 & -0.590 & -0.649 \\
Peso de grano & n.s. & 0.232 & n.s. & n.s. \\
Elongación vegetativa & n.s. & 0.297 & n.s. & n.s. \\
Temperatura foliar & n.s. & -0.353 & n.s. & n.s. \\
Enrollamiento foliar & n.s. & n.s. & n.s. & n.s. \\
Senescencia foliar & n.s. & n.s. & n.s. & n.s. \\
Angulo de la hoja & n.s. & n.s. & n.s. & n.s. \\
Tamaño de la espiga & n.s. & n.s. & n.s. & n.s. \\
Clorofila & 0.297 & 0.314 & n.s. & n.s. \\
Altura de planta & n.s. & n.s. & n.s. & n.s. \\
Foto-oxidación & - & - & n.s. & n.s. \\
Potencial hídrico matutino & - & - & n.s. & n.s. \\
& & & & \\
(B) Intervalo de Floración (IF) & & & n.s. & n.s. \\
Elongación vegetativa & n.s. & n.s. & n.s. \\
Temperatura foliar & n.s. & n.s. & n.s. & n.s. \\
Enrollamiento foliar & n.s. & n.s. & n.s. & n.s \\
Senescencia foliar & n.s. & n.s. & n.s. & n.s \\
Angulo de la hoja & n.s. & n.s. & n.s. & n.s \\
Clorofila & n.s. & n.s & n.s & n.s \\
Potencial hídrico matutino & n.s. & n.s. & n.s. & n.s \\
\hline
\end{tabular}

Nota: Las correlaciones son entre valores promedios de las entradas, y los valores mostrados son solamente para significancia de $\mathrm{P}<0.001$, n.s. indicando no significativo.

La ausencia de correlación entre estas características morfológicas y fisiológicas y el rendimiento de los genotipos bajo sequía pudiera estar relacionada con el uso de parcelas de un solo surco en los viveros. Características que confieren una ventaja comparativa en parcelas de un solo surco bajo sequía como un sistema radicular agresivo, un crecimiento \% rápido, buena altura de planta y hojas laxas, pueden no mostrar su ventaja 
comparativa en parcelas grandes con bordos adecuados. Asimismo, características que confieren ventajas comparativas en parcelas con bordo adecuado pudieran no mostrarlas en parcelas de un solo surco. Sin embargo, quizá sea ventajoso seleccionar estas características como parte de un ideotipo conceptualizado de la tolerancia a la sequía , a pesar de la falta de correlación con el rendimiento de grano bajo sequía en los viveros de selección.

\section{Relación entre el intervalo de la floración y sequía}

Los déficits hídricos impuestos por condiciones sequía retrasan la extrusión de los estigmas en todas las poblaciones de maíz examinadas (Figura 1 A, B, C, D), incrementando el intervalo de la floración, ya que la sequía casi no afecta la antesis. Bajo condiciones normales, todas las famillas (S1 o hermanos completos) tienen relativamente buena sincronización floral, con un intervalo de floración promedio de 2-3 días y un rango máximo cerca de 10 días. Sin embargo, en condiciones de sequía, la extrusión de estigmas se retarda en muchas familias, incrementado el intervalo de la floración. En 1987 el intervalo de la floración promedio en los Pool 16 y 18 fue cerca de 4 días, con un error estándar de 4 días. En 1988, el manejo de la sequía fue más severo para las poblaciones tardías, y el intervalo de la floración promedio para La Posta y el Pool 26 fue de 28 días, con un error estándar de 15 días. Por lo tanto, la sequía permite identificar más fácilmente las familias capaces de sincronizar la floración masculina y femenina, ya que en buenas condiciones la variabilidad genética del intervalo de la floración se reduce considerablemente. La alta densidad poblacional también retrasa la emergencia de los estigmas, pero en menor magnitud que la sequía (Dow et al. 1984). La alta densidad pudiera usarse para seleccionar genotipos superiores en su capacidad de sincronizar la floración masculina y femenina en ausencia de condiciones de sequía.

Relación entre el intervalo de la floración, rendimiento de grano y número de granos

Los datos de los tres niveles de sequía fueron combinados para graficar la relación entre rendimiento de grano y el intervalo de la floración para las 4 poblaciones estudiadas (Figura 2A, B, C, D). El rendimiento mostró tener una relación curvilinear con el intervalo de la floración, decreciendo rápidamente a medida que el intervalo de la floración aumentaba. En todas las poblaciones estudiadas, el rendimiento disminuye aproximadamente $10 \%$ por cada día de retraso en la extrusión delos estigmas desde 0 hasta 9 días. El rendimiento es prácticamente cero cuando el intervalo de la floración excede los 10 días. Esta relación entre el intervalo de la floración y el rendimiento se ha observado en todo el germoplasma examinado hasta ahora, incluyendo co-

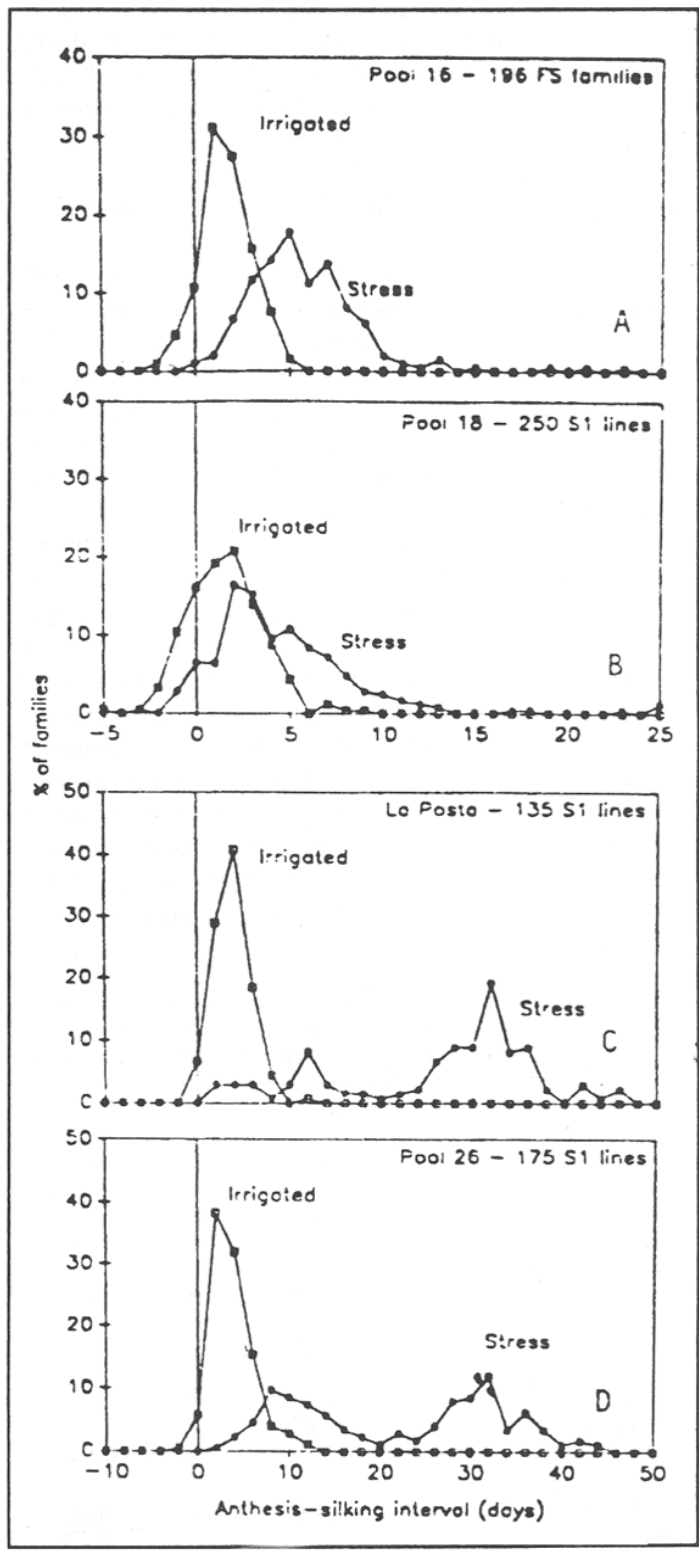

Figura 1. Distribución del intervalo de floración (ASI) bajo riego normal y sequía para 4 poblaciones élite de maíz tropical.

lecciones sin mejoramiento del Banco de Germoplasma de CIMMYT, poblaciones y variedades mejoradas, líneas endocriadas (S1, S2, S3,...S6) e incluso híbridos.

El número de granos por planta tuvo una relación casi idéntica que el rendimiento de grano con el intervalo de la floración (Figura 3A. B, C, D). Esto implica que la pérdida de rendimiento ocurre fundamentalmente debido a un menor número de granos por planta. La relación entre el número de granos por planta y el intervalo de la floración es obvia, ya que cada grano se origina de cada estigma polinizado. Por lo tanto, una gráfica entre el número de granos por planta y el rendimiento es altamente lineal $\left(r^{2}>0.95, \mathrm{P}<0.001\right)$ (datos no mostrados).

Evidencia adicional de la importancia $\mathrm{M}$ intervalo de la floración viene de la evaluación del progreso obtenido 
en la población tropical Tuxpeño Sequía después de 8 ciclos de selección recurrente para resistencia a sequía (Bolaños y Edmeades, 1989, este Congreso). Los datos indicaron que el único progreso significativo registrado fue en reducir progresivamente el Intervalo de la floración bajo sequía, que resultó en un mayor número de granos y mazorcas por planta, y por tanto, un mayor rendimiento de grano. No se registró mejoría alguna ni en el balance hídrico del cultivo o en los otros parámetros fisiológicos y morfológicos indicadores de un balance hídrico positivo. Los datos sugieren asimismo, que las diferencias en el intervalo de la floración son el resultado de diferencias genéticas en los patrones de distribución de materia seca hacia el jilote en desarrollo.

\section{Coeficientes de heredabilidad y correlación genética}

Coeficientes de heredabilidad en el sentido amplio $\left(\mathrm{h}^{2}\right)$ para rendimiento $\left(\mathrm{h}^{2} \mathrm{R}\right)$ y el intervalo de la floración ( $\left.\mathrm{h}^{2} \mathrm{IF}\right)$ y las correlaciones genéticas $(r \mathrm{~A})$ entre ambos fueron computados para las 4 poblaciones en base al análisis de varianza (Hallauer y Miranda, 198 1). La heredabilidad del rendimiento casi no fue afectada por la sequía, hasta que el nivel de rendimiento se redujo en más de $90 \%$, por lo que h2R fue similar para los viveros de riego normal y estrés intermedio. Bajo condiciones de estrés severo, donde el rendimiento se redujo en

Cuadro 2. Heredabilidad en sentido amplio $\left(\mathrm{h}^{2}\right)$ para rendimiento de grano $\left(\mathrm{h}^{2} \mathrm{R}\right)$ y el intervalo de floración ( $\left.\mathrm{h}^{2} \mathrm{IF}\right)$ y las correlaciones genéticas ( $r$ A) entre ambas en 4 poblaciones élite de maíz tropical del CIMMYT cultivadas en tres niveles de sequía en Tlaltizapán, México, en 1987 y 1988.

\begin{tabular}{lcccc}
\hline & $\begin{array}{c}\text { Pool 16 } \\
\text { 194 fam } \\
\text { h.c. }\end{array}$ & $\begin{array}{c}\text { Pool 18 } \\
\text { 250 fam } \\
\mathbf{S}_{\mathbf{1}}\end{array}$ & $\begin{array}{c}\text { La Posta } \\
\text { 232 fam } \\
\mathbf{S}_{\mathbf{1}}\end{array}$ & $\begin{array}{c}\text { Pool 26 } \\
\mathbf{2 3 5} \text { fam } \\
\mathbf{S}_{\mathbf{1}}\end{array}$ \\
\hline $\mathrm{h}^{2} \quad$ Rendimiento & & & & \\
$\quad$ Riego normal & 0.470 & 0.590 & 0.550 & 0.660 \\
$\quad$ Estrés intermedio & 0.280 & 0.610 & 0.690 & 0.590 \\
$\quad$ Estrés severo & 0.380 & 0.540 & 0.410 & 0.390 \\
$\mathrm{~h}^{2} \quad$ Intervalo de floración & & & & \\
$\quad$ Riego normal & 0.360 & 0.690 & 0.640 & 0.730 \\
$\quad$ Estrés intermedio & 0,470 & 0.660 & 0.550 & 0.440 \\
$\quad$ Estrés severo & 0.320 & 0.600 & 0.3 .50 & 0.490 \\
$r$ A Correlación genética & & & & \\
$\quad$ Riego normal & -0.090 & -0.300 & -0.400 & -0.540 \\
$\quad$ Estrés intermedio & -0.170 & -0.450 & -0.510 & -0.770 \\
$\quad$ Estrés severo & -0.830 & -0.680 & -0.850 & -1.010 \\
Rendimiento de grano (ton/ha) & & & \\
Riego normal & 3.501 & 2.355 & 2.873 & 2.778 \\
Estrés intermedio & 1.213 & 1.143 & 1.642 & 1.440 \\
Estrés severo & 0.636 & 0.574 & 0.078 & 0.113 \\
Intervalo de floración (días) & & & \\
Riego normal & 1.170 & 1.140 & 2.740 & 3.100 \\
Estrés intermedio & 2.110 & 1.480 & 2.670 & 5.210 \\
Estrés severo & 5.310 & 4.220 & 35.610 & 28.350 \\
\hline
\end{tabular}

NOTA: Un coeficientes de heredabilidad y correlación genética fueron estimados basados en Hallauer y Miranda (1981).

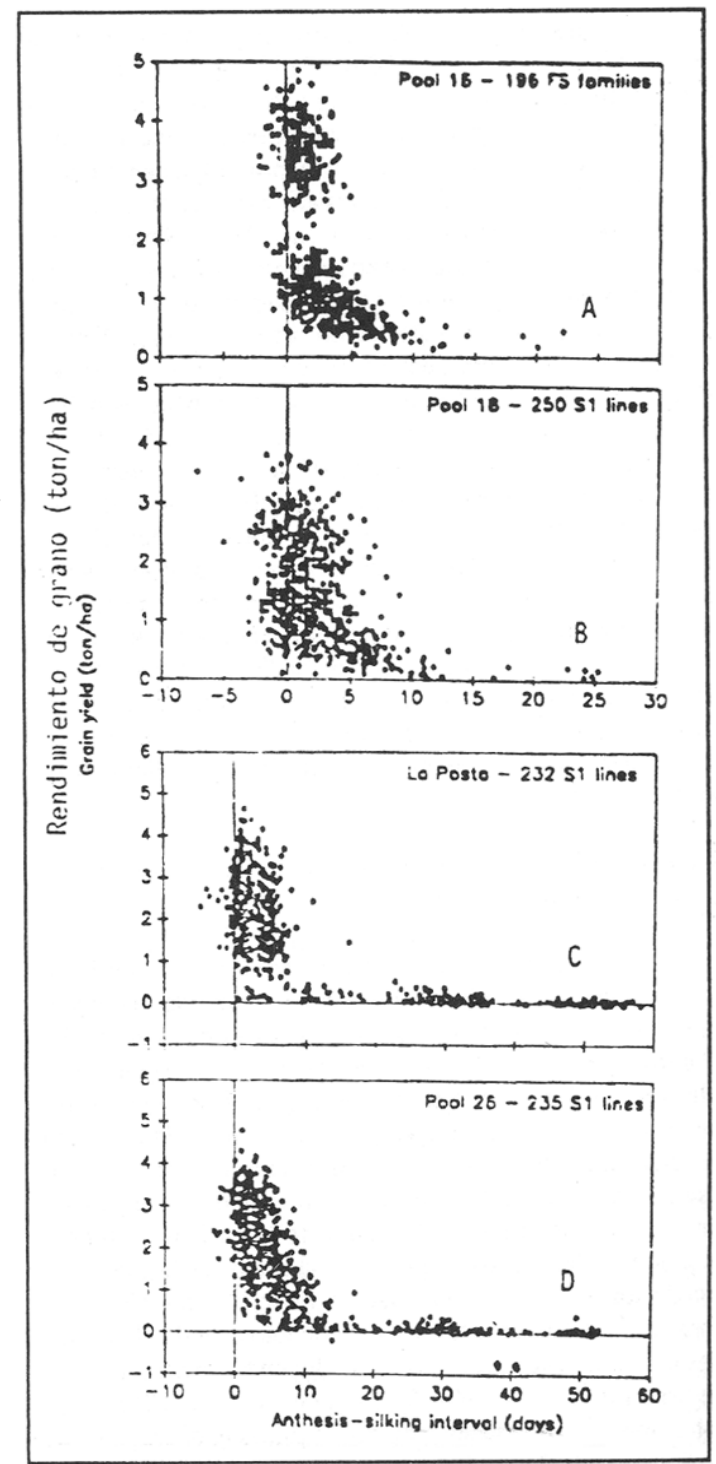

Figura 2. Relación entre el intervalo de la floración (ASI) y rendimiento de grano $(\mathrm{t} / \mathrm{ha})$ para cuatro poblaciones élite de maíz tropical.

más de $80 \%$ en Pool 16 y Pool 18, h ${ }^{2} \mathrm{R}$ fue solamente un poco menor que en las otras dos localidades. Sin embargo, en La Posta y el Pool 26 bajo sequía severa, donde el rendimiento se redujo en más de $90 \%, \mathrm{~h}^{2} \mathrm{R}$ bajó considerablemente (Cuadro 2). Estos datos sugieren que las heredabilidades de rendimiento para estas poblaciones solamente se reducen cuando el rendimiento cae más de $90 \%$. En todos los viveros bajo tres niveles de sequía, $h^{2} \mathrm{R}$ y h2IF fueron menores para familias de hermanos completos que para familias $S_{1}$ 's y $h^{2}$ IF fue menor que $h^{2} \mathrm{R}$ (Cuadro 2). Esto sugiere que es más eficaz usar un sistema de selección basado en líneas $\mathrm{S}_{1}$ que en familias de hermanos completos.

Para la mayoría de las poblaciones y la mayoría de los niveles de estrés, la selección directa para rendimiento de grano resultará en un avance más rápido. Sin 


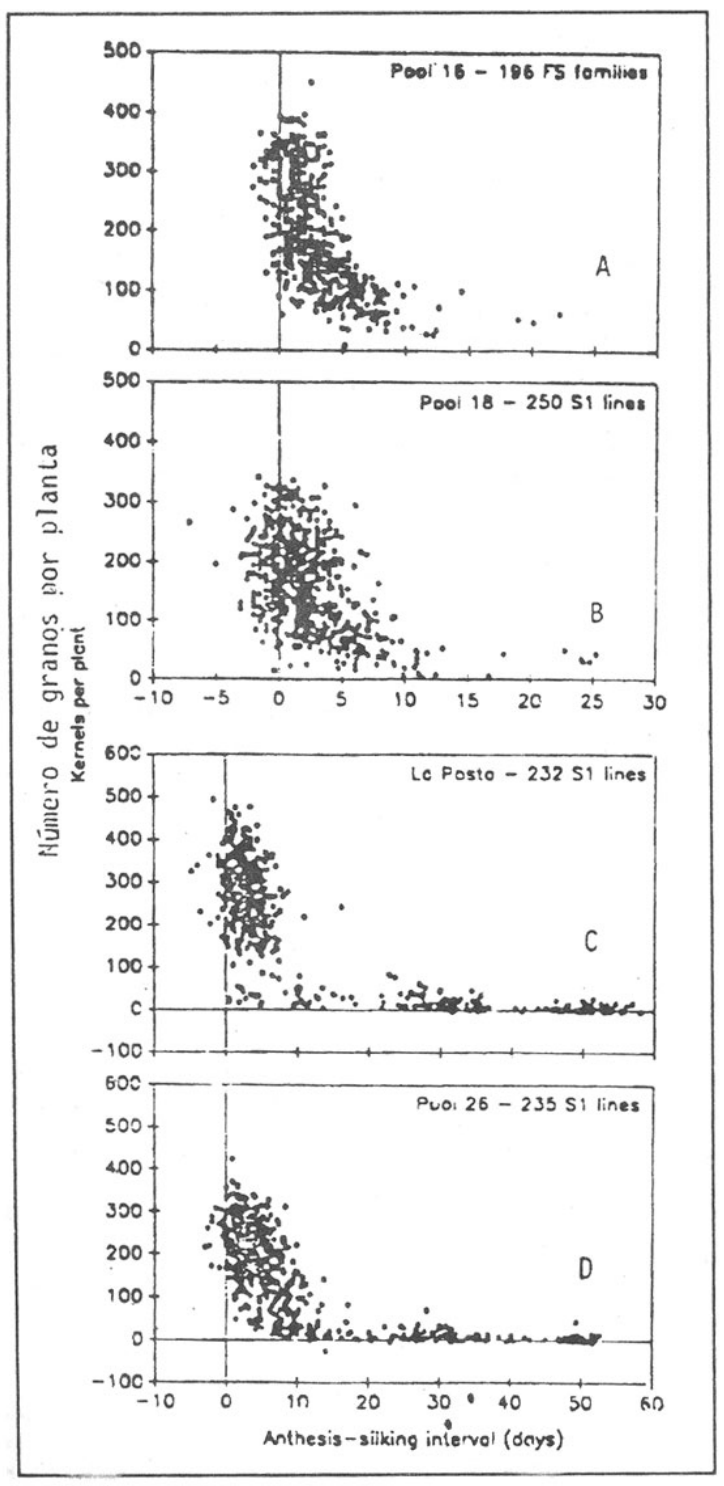

Figura 3. Relación entre el número de granos por plantas y el intervalo de la floración (ASI) para 4 poblaciones élite de maíz tropical.

embargo, para el Pool 26 bajo sequía severa, cuando la heredabilidad del rendimiento es baja, $r \mathrm{~A} * \mathrm{hIF}>\mathrm{hR}$, lo que muestra que las ganancias esperadas en rendimiento al seleccionar para un intervalo de floración reducido serán mayores que seleccionando para rendimiento. $\mathrm{Pa}$ ra todas las poblaciones estudiadas, la correlación genética $(r \mathrm{~A})$ entre el intervalo de la floración y rendimiento incrementó a medida que el nivel de estrés aumentó, mostrando el valor selectivo de este parámetro secundario relativamente fácil de medir.

\section{Datos de las variedades experimentales de las fracciones superior e inferior de la población}

Para estimar el valor adaptivo de parámetros de selección y heredabilidades realizadas, variedades experi- mentales (VE) rutinariamente se forman en esquemas divergentes entre las 10 mejores y peores familias para cada característica de cada población en mejoramiento para resistencia a sequía. Normalmente se forman las siguientes VE: (a) El mejor rendimiento bajo sequía, (b) El mejor rendimiento bajo riego normal, (c) Mejory peorintervalo de floración bajo sequía, (d) Mejor y peor para todas las características (el producto del índice de selección que incorpora todas las características), (e) El mejor y peor para elongación relativa foliar, (f) Mejor y peor temperatura foliar, (g) Hojas erectas versus hojas laxas, (h) Mejor y peor senescencia o fogueo foliar. Estas VE se comparan con la población para estimar su valor adaptivo dentro del esquema de mejoramiento y su heredabilidad realizada.

El Cuadro 3 muestra datos de rendimiento de grano, bajo riego normal y sequía para las VE del Pool 26 evaluadas en Tlaltizapán en 1988. Los resultados muestran que la VE formada basada en todas las características deseables (el índice de selección que incorpora todos los parámetros) rinden significativamente mejor que la VE de todas las características indeseables bajo riego normal y sequía. La VE formada en base al rendimiento bajo condiciones de riego normal también se comportó mejor solamente en buenas condiciones. Ninguna diferencia significativa se encontró entre las VE formadas de las mejores y peores familias para temperatura foliar, elongación relativa foliar, senescencia foliar, hojas erectas y laxas. Estas características morfológicas y fisiológicas en sí parecen contribuir muy poco al progreso en la resistencia a sequía. Sin embargo, la VE

Cuadro 3. Rendimiento de grano $(\mathrm{kg} / \mathrm{ha})$ de variedades experimentales (VE), del Pool 26 bajo riego normal y sequía en Tlaltizapán, 1988.

\begin{tabular}{lcr}
\hline \multicolumn{1}{c}{ Variedad experimental } & \multicolumn{2}{c}{ Rendimiento (kg/ha) } \\
& Riego normal & Sequía \\
\hline Pool 26 Ciclo 0 & 4934 & 1582 \\
Mejor en "todas" características & 5188 & 2370 \\
Peor en "todas" características & 3722 & 598 \\
Mejor rendimiento bajo sequía & 4589 & 1997 \\
Mejor rendimiento bajo irrigación & 5137 & 10773 \\
Mejor intervalo de floración & 4670 & 1588 \\
Peor intervalo de floración & 3503 & 862 \\
Mejor elongación vegetativa & 4225 & 1477 \\
Peor elongación vegetativa & 4891 & 1737 \\
Mejor senescencia foliar & 4148 & 762 \\
Peor senescencia foliar & 3844 & 1045 \\
Temperatura más baja & 4202 & 1461 \\
Temperatura más alta & 3946 & 1092 \\
Hojas erectas & 4420 & 1577 \\
Hojas laxas & 3696 & 1127 \\
F tratamientos (19, 38 g.1.) & $* *$ & $\mathrm{NS}$ \\
D.M.S. (0.05) & 1067 & 1122 \\
C.V. & 14.9 & 50.1 \\
\hline
\end{tabular}

* Significativo al $0.05 \%$ probabilidad. NS No significativo. 
superior resultó ser la que incluía "todas" las características deseables. Estos datos, a pesar de ser aún inconclusos, muestran que se puede lograr un progreso más rápido usando ciertos criterios morfológicos y fisiológicos, además del rendimiento.

El Cuadro 4 muestra los datos de las VE en cuanto al comportamiento de las características morfológicas y fisiológicas y permite estimar las heredabilidades realizadas de fogueo foliar, temperatura foliar ángulo de la hoja (erecta contra laxa) y elongación vegetativa. Para las características relacionadas con senescencia de las hojas, como las calificaciones visuales de fogueo, calificaciones de foto-oxidación y mediciones del contenido de clorofila en las hojas, los datos muestran que, son características con relativa alta heredabilidad. Lo mismo sucede con el ángulo de la hoja (erecta contra laxa). En los tres casos, la VE formada por las 10 mejores familias resultó su significativamente mejor en estos criterios que el $\mathrm{C} 0$ de la población y que las 10 peores. Para las características de temperatura foliar y elongación vegetativa foliar, los datos muestran que casi no tienen heredabilidad, ya que la VE formada por las mejores 10 familias se comportó peor que la VE formada por las peores $10 \mathrm{y}$ que el ciclo 0 . Dicho de otro modo, la variabilidad observada en los viveros para elongación vegetativa y temperatura foliar es, en su mayoría, ambiental y no genética, y por lo tanto no vale la pena su uso como criterio de selección.

Cuadro 4. Comportamiento de VE del Pool 26 formados en base a características fisiológicas y morfológicas bajo riego normal y sequía en Tialtizapán, México en 1988

\begin{tabular}{lrrrrr}
\hline \multicolumn{1}{c}{ Característica } & Ciclo 0 & Mejor & Peor & F(tmt) & $\begin{array}{l}\text { DMS } \\
(5 \%)\end{array}$ \\
\hline Calif de fogueo foliar & 4.67 & 4.04 & 5.54 & $* * *$ & 0.586 \\
Calif. de foto-oxidación & 3.83 & 3.33 & 4.41 & $* * *$ & 0.625 \\
Clorofila (m/cm2) & 56.96 & 59.96 & 54.36 & $* * *$ & 4.130 \\
Calif. de hoja erecta & 3.00 & 2.56 & 3.33 & NS & 0.586 \\
Temperatura foliar (C) & 0.86 & 0.52 & 0.22 & NS & 1.776 \\
Elongación vegetativa (\%) 57.90 & 53.89 & 60.91 & NS & 5.823 \\
\hline
\end{tabular}

*** Significativo a $\mathrm{P}<0.001$

NS No significativo al $\mathrm{P}<0.001$

Calif. de fogueo foliar en escala de 1-9, 1 siendo más verde Calif. de foto-oxidación en escala de 1-9, 1 siendo más verde Clorofila en $\mathrm{ug} / \mathrm{cm}^{2}$

Calif. de hoja erecta en escala de 1-5, 1 siendo erecta y 5 laxa Temperatura foliar expresa la diferencia entre hoja y aire en $\mathrm{C}$ Elongación vegetativa en sequía como \% del valor en riego normal

\section{CONCLUSIONES}

1. Existe suficiente variabilidad genética para rendimiento, el intervalo de la floración, y para parámetros fisiológicos y morfológicos indicativos de resis- tencia a sequía en el germoplasma élite de maíz tropical del CIMMYT. El estrés se debe manejar para permitir la máxima expresión de esta variabilidad presente ya niveles representativos de los ambientes, para los cuales el material se encuentra destinado.

2. La sequía afecta el rendimiento del maíz reduciendo el número de granos y mazorcas por planta, fundamentalmente debido a un retraso en el intervalo de la floración y una pérdida de la viabilidad de los estigmas Esta mayor variabilidad en el intervalo de la floración bajo sequía permite una identificación más fácil de las familias superiores.

3. Características morfológicas y fisiológicas supuestamente indicativas de un buen balance hídrico parecen tener un uso limitado en programas de mejoramiento de maíz tropical, por lo menos en los estadios iniciales. El rendimiento de las líneas bajo sequía parece estar limitado por la capacidad de la línea en producir estigmas viables un intervalo de floración reducido.

4. La evaluación de VE formadas mediante selección divergente indica, sin embargo, que el uso de estas características con supuesto valor adaptivo puede resultar en progreso más rápido que el uso de rendimiento 0 el intervalo de floración solamente. En particular, seleccionar para senescencia foliar lenta prolongando la intercepción activa de radiación durante el llenado de grano y para hojas erectas son buenos criterios de selección. Esto ocurre a pesar de una ausencia de correlación entre estas características y el rendimiento bajo sequía.

5. La estrategia actual del CIMMYT para mejorar la resistencia a sequía del maíz tropical es seleccionar primero para buena sincronización floral y rendimiento bajo sequía, asegurándose de mantener el potencial productivo y la madurez del germoplasma constante. Seleccionar para otras características puede ser útil después que la población haya sido mejorada para la sincronización floral.

\section{BIBLIOGRAFIA}

BOLAÑOS, J.; EDMEADES, G.O. 1989. Cambios en la población Tuxpeño sequía después de ocho ciclos de mejoramiento para resistencia a sequía. In: Reunión Anual del PCCMCA (35,1989, San Pedro, Sula, Honduras.

DOW, E.W.; DAYNARD, T.B.; MULDOON, J.F.; MAYOR, DJ.; THURTELL, G.W. 1984. Resistance to drought and density stress in Canadian and European maize (Zea mays L.) hybrids. Can. J. Plant Sci. 64:575-585.

HALLAUER, A.R.; MIRANDA, J.B. 1981. Quantitative genetics in maize breeding. Ames, Iowa State University. 